\title{
New Adaptive Method to Optimize the Secondary Reflector of Linear Fresnel Collectors
}

\author{
Guangdong Zhu \\ Senior Engineer, Ph.D., National Renewable Energy Laboratory, 15013 Denver West Parkway, Golden, \\ CO 80401 USA, Guangdong.Zhu@nrel.gov
}

\begin{abstract}
Performance of linear Fresnel collectors may largely depend on the secondary-reflector profile design when small-aperture absorbers are used. Optimization of the secondary-reflector profile is an extremely challenging task because there is no established theory to ensure superior performance of derived profiles. In this work, an innovative optimization method is proposed to optimize the secondary-reflector profile of a generic linear Fresnel configuration. The method correctly and accurately captures impacts of both geometric and optical aspects of a linear Fresnel collector to secondary-reflector design. The proposed method is an adaptive approach that does not assume a secondary shape of any particular form, but rather, starts at a single edge point and adaptively constructs the next surface point to maximize the reflected power to be reflected to absorber(s). As a test case, the proposed optimization method is applied to an industrial linear Fresnel configuration, and the results show that the derived optimal secondary reflector is able to redirect more than $90 \%$ of the power to the absorber in a wide range of incidence angles. The proposed method can be naturally extended to other types of solar collectors as well, and it will be a valuable tool for solar-collector designs with a secondary reflector.
\end{abstract}

\section{Introduction}

Linear Fresnel is one of four major concentrating solar power (CSP) technologies, along with parabolic trough, power tower, and dish/engine. As a type of line-focus technology, a linear Fresnel collector is typically composed of an array of one-axis primary reflectors and a fixed receiver assembly. The primary reflectors track the sun and focus sunlight to the receiver assembly. The receiver assembly includes one or multiple absorber tubes and an optional secondary reflector. In the past, linear Fresnel technology was treated as a low-cost, low-concentration option to produce thermal energy at a low or intermediate temperature [1]. Lately, the CSP community has begun to recognize its potential to achieve high concentrations and high temperatures [1-3]. Commercial direct-steam-generation (DSG) linear Fresnel products exist that can produce steam with a temperature as high as $450{ }^{\circ} \mathrm{C}[4,5]$, and high-temperature molten-salt linear Fresnel collectors (up to $550^{\circ} \mathrm{C}$ ) are also an active research topic and under development [6-10]. The superior feature of molten salt over water/steam is that it not only provides a high-temperature low-vapor-pressure heat transfer fluid (HTF) in the solar field, but also, a low-cost thermal storage media for a utility-scale electricity system using linear Fresnel technologies.

The secondary reflector as part of receiver assembly is used to improve collector performance and - in the cases when non-evacuated absorbers are used - to reduce heat loss of absorbers. Examples of linear Fresnel secondary-reflector shapes include trapezoidal, parabolic, or other profiles defined by a higherorder polynomial, as well as compound parabolic shapes [4, 10-13]. The trapezoidal shape provides a fabrication-friendly engineering design and facilitates the addition of an insulation layer to reduce heat 
loss from non-evacuated absorbers $[11,14]$. The parabolic shape can naturally concentrate the incoming parallel sun rays to its focal point [10], but the reflected light from the primary reflector is not parallel, thus leading to non-ideal optical performance. A higher-order polynomial will provide more design parameters to optimize the secondary profile, but this often requires more intensive computational efforts.

One popular secondary-reflector shape resembles a type of non-tracking collector designs - compound parabolic concentrators (CPC) $[15,16]$. The CPC design is a type of non-tracking solar thermal collector that directly accepts sunlight. In contrast, a linear Fresnel secondary reflector accepts reflected light from primary reflectors, which results into two major differences: 1) the acceptance angle for the secondary reflector is required to cover the whole primary reflector field, and 2) the reflected sun shape becomes widened when reaching the secondary reflector because of the relative long optical path and system optical errors. Thus, the CPC cannot be justified as the optimal solution for a linear Fresnel secondary reflector.

The optimal secondary-reflector profile should be able to achieve the highest optical performance and it will vary with different collector configurations. In this work, a generic optimization methodology is proposed to derive optimal secondary-reflector profiles for a solar collector. Linear Fresnel is selected to illustrate the methodology, but the proposed method can be applied to both line-focus and point-focus collectors. It can also be extended to stationary solar collectors.

The paper is organized as follows: Section II briefly describes key linear Fresnel aspects; Section III presents the proposed optimization methodology in detail; Section IV illustrates the value of the proposed method by selecting an industrial linear Fresnel collector design as a test case; lastly, Section V discusses the work presented and its future potential. 


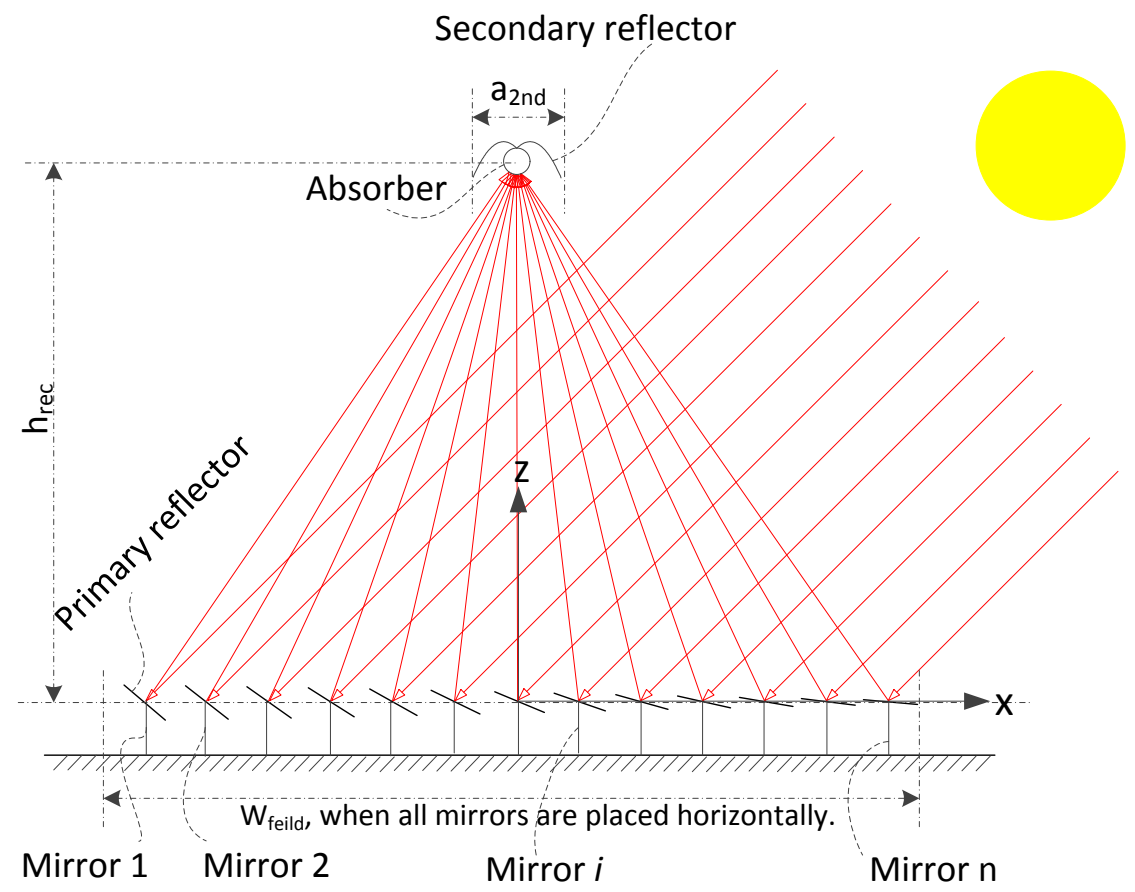

(a): Geometry

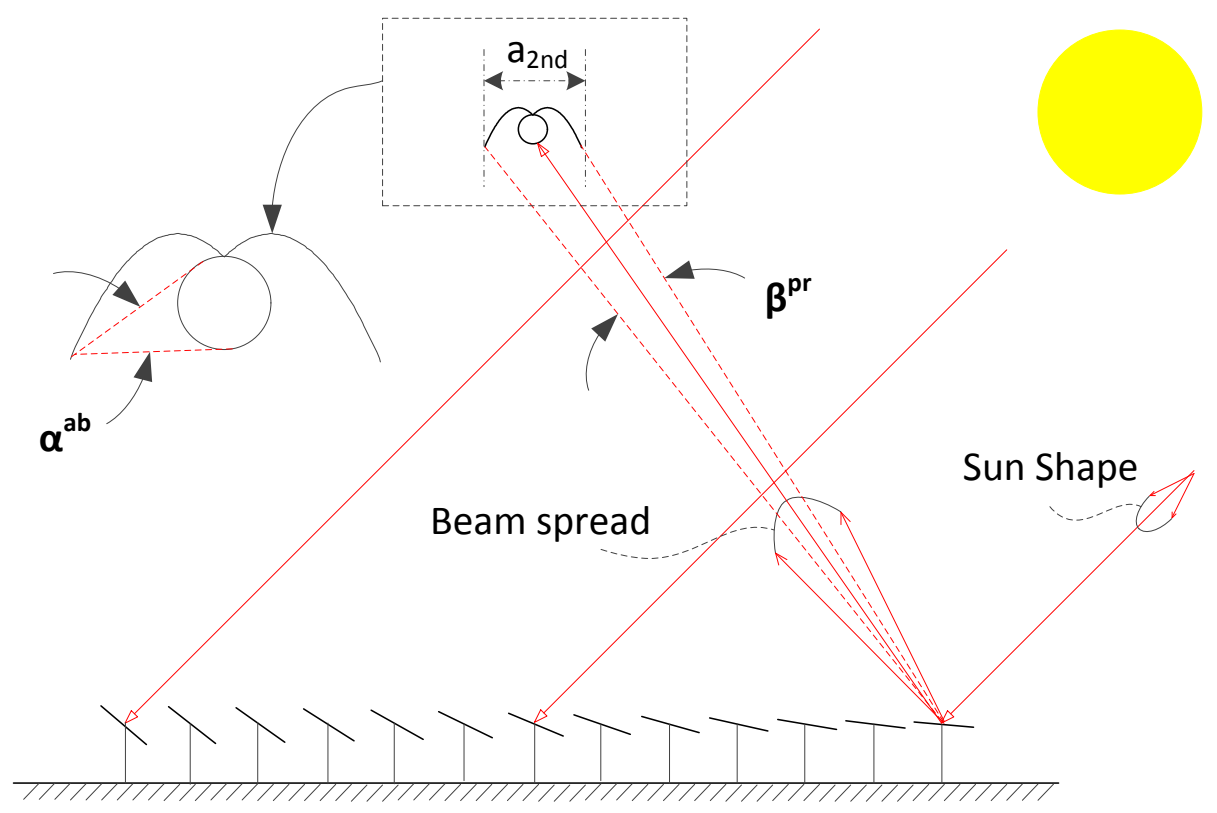

(b): Optics

Figure 1: Linear Fresnel collector characteristics: geometry and optics. For the sake of illustration, the sun size, secondary reflector, and absorber are not scaled relative to the size of primary reflectors.

\section{Linear Fresnel Aspects}

As shown in Fig. 1, a linear Fresnel collector is characterized by its geometry and system optics. With respect to its geometry, the collector includes an array of primary reflectors and a receiver assembly with 
a secondary reflector. Primary reflectors track the sun and reflect sunrays to the absorber either directly or indirectly through a secondary reflector.

With respect to system optics, various optical error sources exist such as imperfect mirror specularity, primary mirror slope error, primary mirror tracking error, absorber position error, and associated errors on secondary reflectors. In particular, mirror specularity describes the phenomenon that the reflected light accumulates around the specular direction defined by the law of reflection on a mirror surface. A perfect specularity denotes the situation that the $100 \%$ of reflection power is reflected along the specular direction. In reality, there always exist some degree of imperfection in the microscopic texture of reflecting surfaces and thus mirror specularity is always destroyed accordingly $[17,18]$. The effective impact of all system optical errors to the original sun shape is to broaden the reflected sun ray beam. The broadened beam after reflection may be much wider than the original sun shape; when it reaches the receiver assembly, the reflected light lying within the acceptance-angle window is intercepted by the receiver-assembly aperture.

The root mean square (RMS) of the overall beam-spread distribution can be estimated by assuming that the sun shape and all system optical errors follow a Gaussian distribution [19]:

$$
\sigma_{\text {total }}^{2}=\sigma_{\text {sun }}^{2}+\sigma_{\text {specularity }}^{2}+\left(2 \sigma_{\text {slope }}\right)^{2}+\left(2 \sigma_{\text {track }}\right)^{2}+\sigma_{\text {receiver_assembly }}^{2}
$$

Here, $\sigma_{\text {sun }}$ is the RMS of the sun shape; $\sigma_{\text {specularity }}, \sigma_{\text {slope }}, \sigma_{\text {track }}$, and $\sigma_{\text {receiver_assembly }}$ are the RMS for mirror specularity, mirror slope error, mirror tracking error, and receiver-assembly-related optical error, respectively. When the sun shape and/or some optical error sources are not a Gaussian distribution, the total beam distribution, RMS $\sigma_{\text {total }}$, can be obtained through convolutions of individual distributions [20, 21], or by use of Hermite functions [22]. The factor of 2 for the slope error and the tracking error is derived from the law of reflection. The optical errors in Equation (1) are independent of the mirror size and can be measured with respect to a reference mirror shape, such as a flat surface or a parabolic surface. For a specific collector design, the size and reference shape of mirror facets will be taken into account as part of the collector geometry when the collector optical performance (intercept factor or optical efficiency) is calculated. Taking the slope error as an example, when flat mirrors are used in a collector design, the slope error can be measured with respect to a flat surface and, in a ray-trace model, the flat mirrors and their associated slope error will be modelled. Beside ray-trace models, there exist analytical methods to calculate the collector performance [20, 21, 23-27].

As derived from the two aspects of linear Fresnel collectors, at any surface point on the primary reflectors, the reflected beam will be able to cover the entire secondary-reflector aperture if not blocked or shaded. Thus, because of beam spread, each surface point on the secondary reflector will receive reflected light (different magnitude of power intensity) from all primary reflector surfaces except the parts that are shaded or blocked. Figure 2 illustrates the incidence angular range for two example points. Point A can see the entire primary-reflector array, so its incidence angular range covers the whole field. For Point B, its incidence angular range covers a partial mirror array because the absorber directly intercepts reflected light. By doing this for the whole surface of the secondary reflector, the central incidence can be readily calculated for each point. The central incidence directions are illustrated for Point A and B in the figure. 
One key observation is that the incidence angular range for a secondary reflector is much wider than the sun shape and the beam-spread combined effects of system optical errors. Assuming that the receiverassembly height is $7 \mathrm{~m}$ and the primary-field width is $14 \mathrm{~m}$, then the incidence angular range for the secondary-reflector edge will be about $45^{\circ}$, whereas the RMS $\left(\sigma_{\text {total }}\right)$ of a broadened beam spread may be in the range of $1^{\circ}-2^{\circ}[7,19]$.

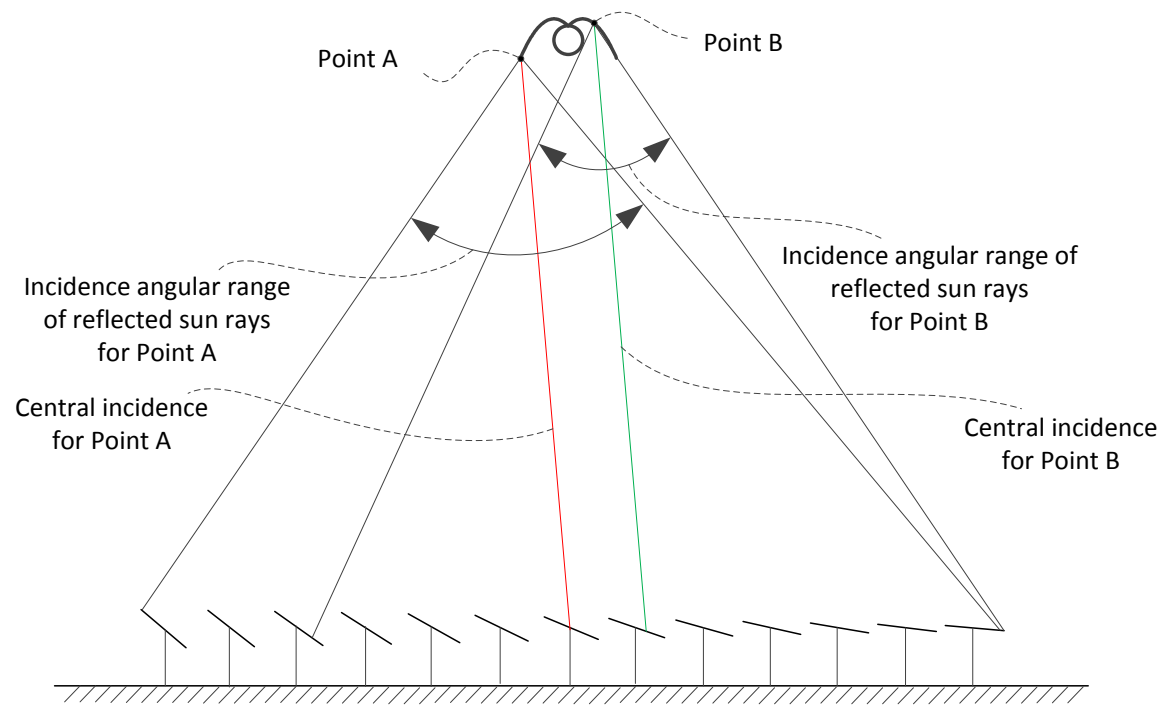

Figure 2: Incidence of reflected sun rays to secondary reflectors.

\section{General Adaptive Optimization Method}

The proposed method does not assume any particular family of analytical curves for secondary-reflector profiles. Instead, it simply starts at a pre-specified edge point and advances into a complete profile in the way that the profile is able to reflect the maximum amount of power/energy to the absorber. The optimal adaptive profile will depend on the specific linear Fresnel configuration such as absorber size and position, and collector-field size.

\section{i. Determination of secondary-reflector aperture}

The secondary-reflector aperture is typically determined through a comprehensive collector optimization. Here, a simplified procedure is provided to determine the secondary-reflector aperture.

For a given linear Fresnel design, assume the secondary-reflector aperture is required to intercept $95 \%$ of reflected power for the outermost mirror $n$. Thus, the acceptance angle for mirror $n$ needs to be:

$$
\beta_{n}^{p r}=4 \cdot \sigma_{\text {total }}
$$

The required secondary-reflector aperture is then:

$$
\begin{gathered}
a_{2 n d} \cong l_{n} \cdot \sin \left(\beta_{n}^{p r}\right), \text { if primary mirrors are parabolic; } \\
a_{2 n d} \cong l_{n} \cdot \sin \left(\beta_{n}^{p r}\right)+w_{n} \cdot \cos \left(\tau_{n}\right), \text { if primary mirrors are flat. }
\end{gathered}
$$

Here, $l_{n}$ is the distance between the $n$-th mirror (the outermost mirror) center and the absorber center; $w_{n}$ is the $\mathrm{n}$-th mirror width; and $\tau_{n}$ is the tracking angle of the $\mathrm{n}$-th mirror at the normal incidence. The 
underlying assumption is that the secondary-aperture width is small compared to the absorber height. The calculation of the secondary-reflector aperture here may vary for different linear Fresnel designs.

\section{ii. Adaptive advancement into an optimal secondary shape}

Assume the secondary-reflector aperture $\left(\mathrm{a}_{2 n d}\right)$ is determined. As an adaptive method, the starting point for the optimal secondary-reflector surface is determined by the aperture width. The height difference between the secondary starting point and the absorber center is defined as $\Delta h$, in Figure 3(a). It will be optimized later.

As illustrated in Figure 3(a), at the starting point, the principal incidence is determined first. Here, the principal incidence is defined as the incidence ray direction that the local secondary surface will reflect to the absorber center and is the optimal direction so that maximum amount of sun ray power will be reflected to the absorber at this point. Strictly speaking, the principal incidence can be determined by performing detailed ray-trace simulation or certain analytical methods. In a simplified approach, the principal incidence can be chosen as the central incidence accounting for the absorber blockage, as illustrated in Figure 2.

The target reflection direction is pointing to the absorber center, by connecting the starting point with the absorber center. Once the principal incidence and the target reflection direction are identified, the surface normal at the starting point will be the one bisecting the principal incidence and the target reflection.

The next step is to determine the next point along the secondary-reflector surface. Along the surface tangent vector, the second surface point is determined by specifying a step distance (depending on the required surface accuracy). Then the principal incidence, the target reflection, the surface normal, and the surface tangent at the second surface point can be determined by repeating the process for the starting point.

For a better surface approximation, an optional iterative process can be taken to update the next surface point: first calculate the angle between the surface-normal vectors at the first two surface points, and then adjust the surface tangent by half of the calculated angle so that the angular difference between the surface normal and the surface tangent are the same for the two neighboring surface points.

The process is repeated to find the remaining surface part and a complete reflector shape is obtained. Figure 3(b) gives an example of a secondary-reflector shape. 


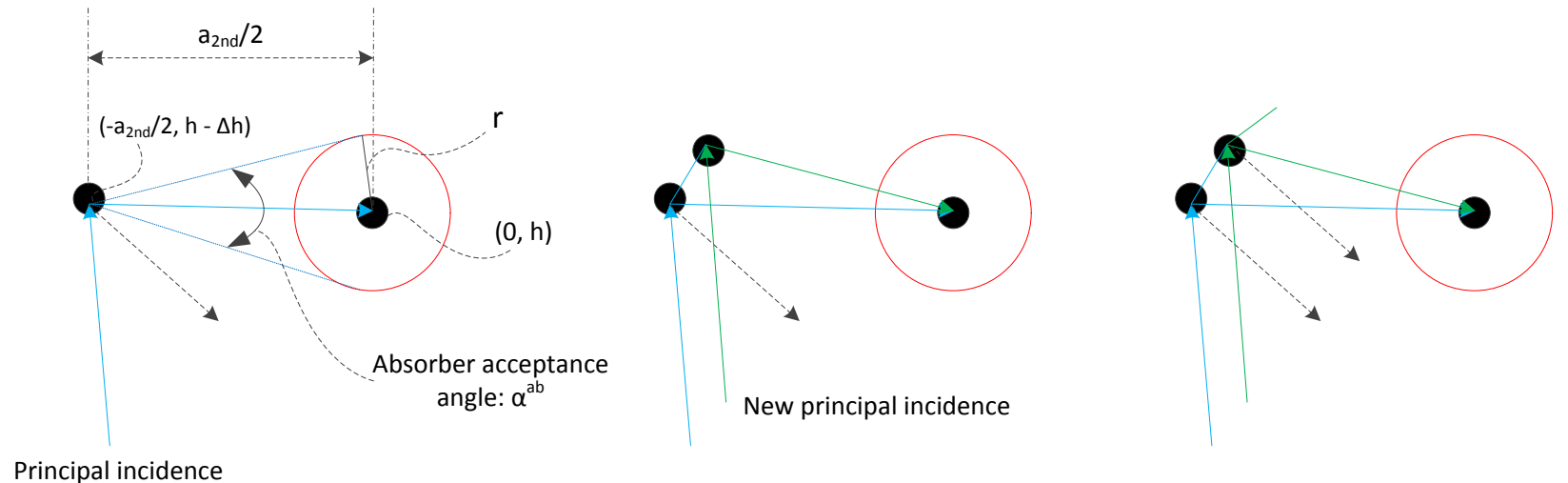

(a): Determination of next optimal point on secondary-reflector surface. Black dots on the side illustrate the first two surface points on the optimal secondary profile for the purpose of illustration.

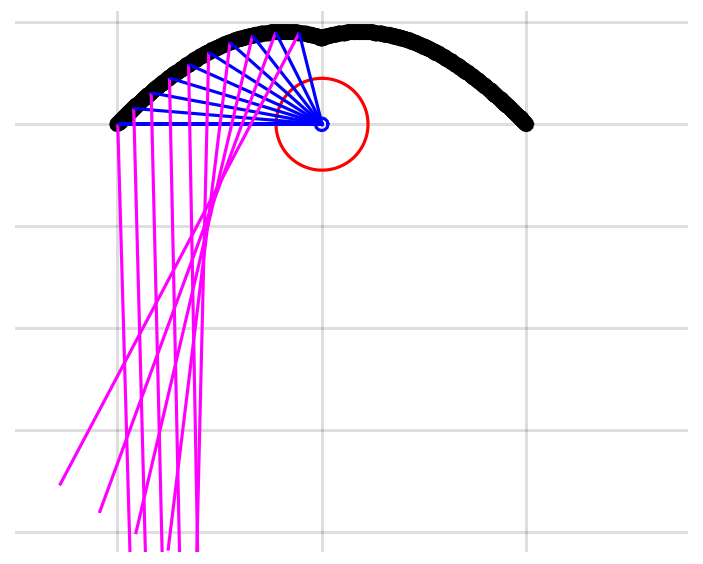

(b): One complete optimized secondary-reflector surface. The pink lines are principal incidence and the blue lines are the target reflection direction. Red circle is the absorber surface and the glass envelope for evacuated receivers is omitted here.

Figure 3: Adaptive optimization of a secondary-reflector surface.

\section{iii. Determination of principal incidence using accumulated power intensity}

Principal incidence at any point along the optimal secondary-reflector surface serves to determine the local surface normal so that most power can be reflected to the absorber. The acceptance angle of the absorber $\alpha^{a b}$ is typically much less than the incidence angular range for a secondary-reflector surface point. Thus, very often, central incidence is not the best approximation for principal incidence. 


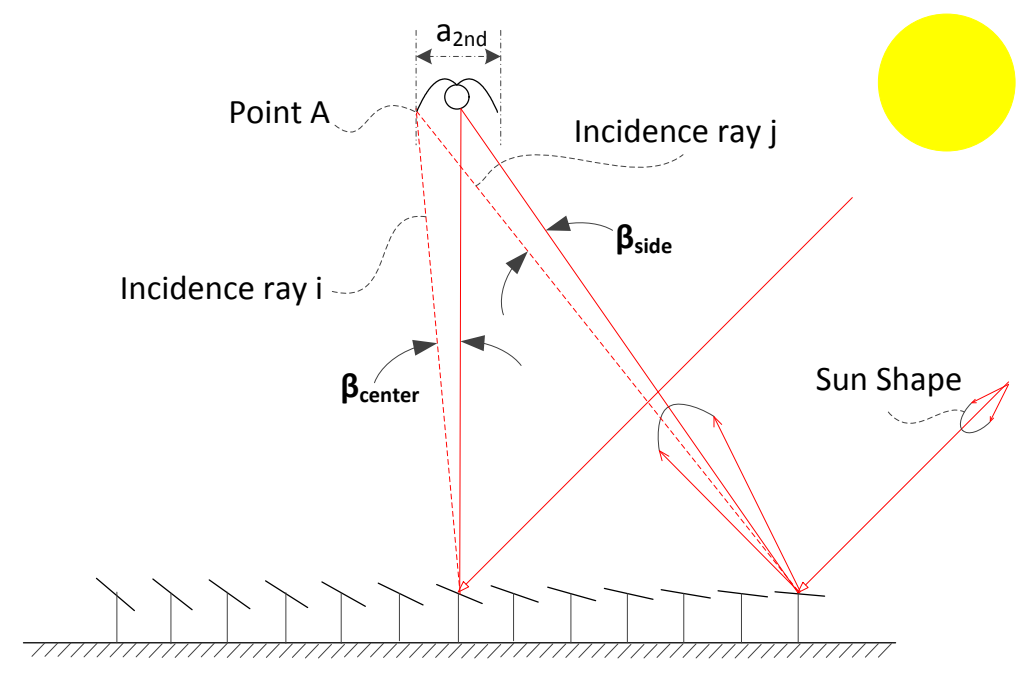

(a): Acceptance angle of secondary-reflector aperture changes with primary-mirror position due to varying optical path between individual mirror and secondary aperture.

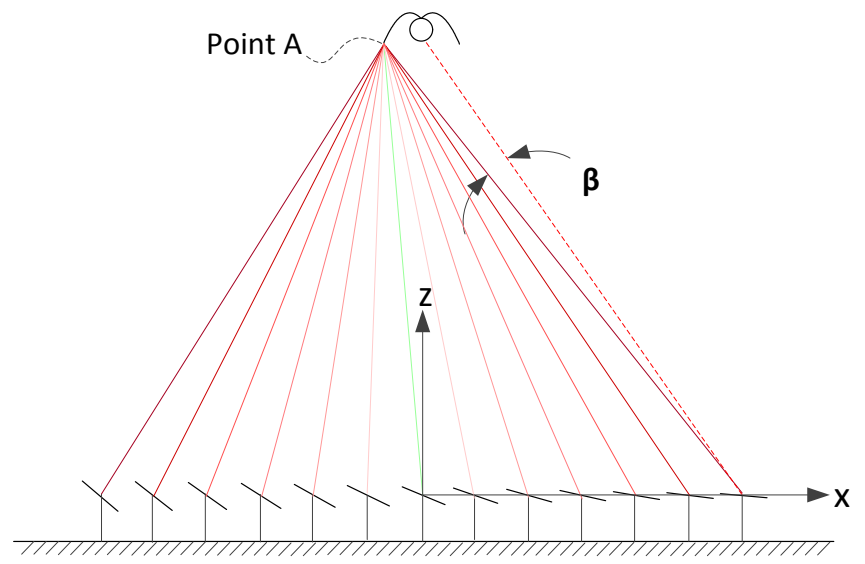

(b): Power density changes with primary-mirror position at Point A because the relative deviation from the nominal tracking direction changes for each primary mirror. The change of the line color indicates the change of power intensity. The green line has the least power intensity. 


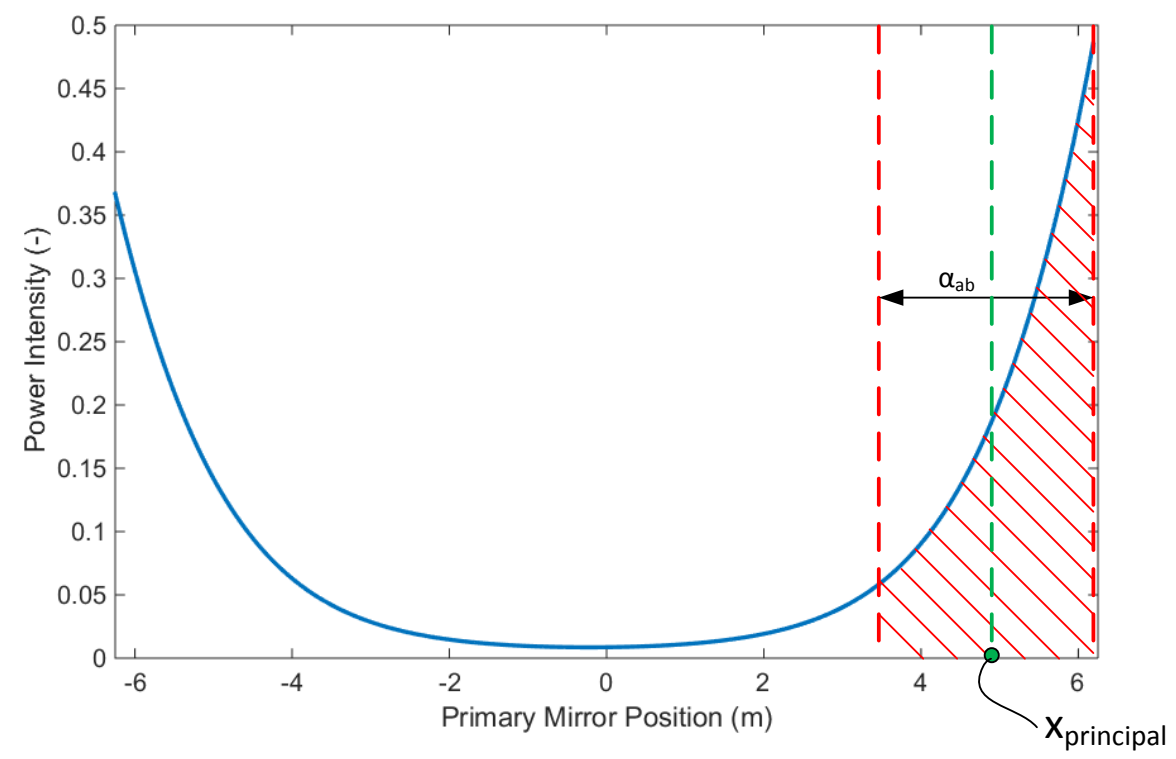

(c): Determination of the principal incidence point using the receiver acceptance angle. Here a collector with a primary field size of about $12 \mathrm{~m}$ is used for the purpose of illustration.

Figure 4: Determination of principal incidence based on accumulated secondary power density.

Here, a more accurate but simple approach is described to determine principal incidence at any point on the secondary surface. First, as illustrated in Figure 4(a), the acceptance angle of the primary reflector is smaller for the mirrors closer to the array center than for the mirrors further away, which can be also considered the deviation angle of Point A from the mirror tracking direction. In the figure, it can be seen that:

$$
\beta_{\text {center }}<\beta_{\text {side }}
$$

Here, $\beta_{\text {center }}$ and $\beta_{\text {side }}$ are the deviation angle for the center mirror and the side mirror with respect to Point A, respectively. This implies that the secondary power intensity for incidence ray $i$ is less than for incidence ray $j$. The secondary power intensity represents the power intensity of incoming reflected sun rays from primary reflectors to a point on the secondary surface. Thus, for Point A on the secondary surface, the secondary power intensity varies with the position of the mirror. As shown in Figure 4(b), secondary power intensity decreases from the left outermost mirror to the center mirror and then increases toward the right outermost mirror. More specifically, the power density of each ray is the function of the deviation angle, which is then a function of mirror position. Assuming that the reflected beam-spread follows a Gaussian distribution, it can be obtained:

$$
P(x)=g\left(\sigma_{\text {total }}, \beta^{p r}(x)\right)
$$


Here, $g$ is a Gaussian function with a standard deviation of $\sigma_{\text {total }}$ and it will give the intensity at a deviation angle of $\beta^{p r}(x)$. As illustrated in Figure 4(b), $x$ is the mirror position along the horizontal direction and $\beta^{p r}(x)$ can be calculated as a function of the mirror position.

Figure 4(c) gives secondary power intensity of the incidence ray as a function of mirror position at the secondary reflector surface point A. As expected, the secondary power intensity first decreases and then increases with increasing distance between the mirror position and the absorber center (the aiming point). However, the secondary power intensity for right-side mirrors is higher than mirrors on the left because the elongated optical path to the right-side mirrors leads to a smaller deviation angle and thus higher secondary power intensity. A window with a size of the absorber acceptance angle $\left(\alpha^{a b}\right)$ for Point A is created to move from left to right so that the principal mirror position can be determined to give rise to the most accumulated power within the absorber acceptance window. If the absorber is large enough, the acceptance-angle window will be larger than the entire mirror array and the central incidence would be the principal incidence.

This process can be repeated for any other points on the secondary surface. The view blockage of the absorber tube will be accounted for when it applies. Figure 5 gives three example optimal secondary profiles that vary with relative secondary height $\Delta h$ and the absorber size. For all cases, starting from the left-edge point of the secondary surface, the principal incidence comes from the other side of primaryreflector field first (i.e., for left-side secondary surface, principal incidence comes from right-side mirrors), and then shifts to the same-side mirrors because of the absorber blockage and the decreasing deviation angle. For the profile in the right plot of Figure 5, the central flat part comes from the fact that a minimum distance is set between the absorber and the secondary because of the existence of a glass envelope when a vacuum absorber is used.
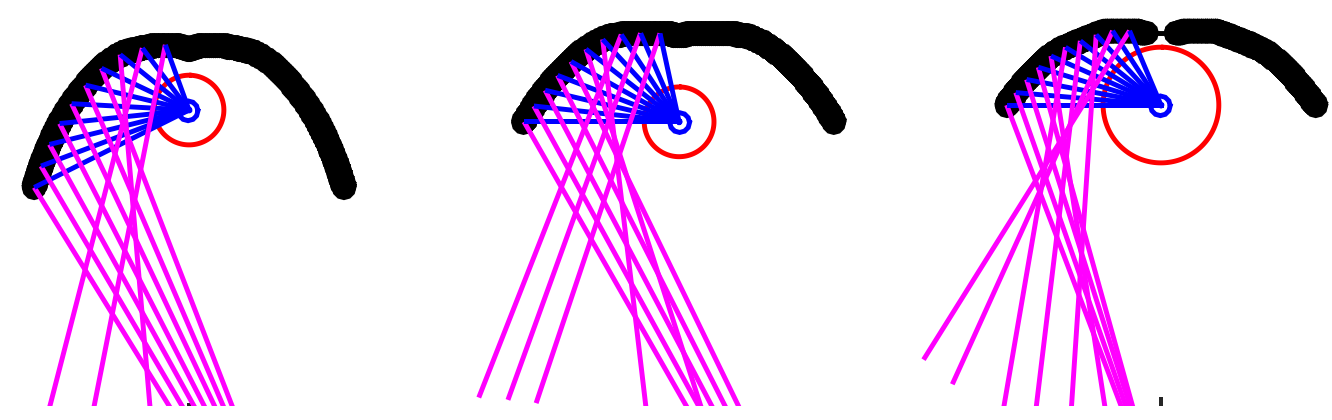

Figure 5: Optimal secondary shapes with different height and absorber size. The pink lines are principal incidence and the blue lines are the target reflection direction. The circle is the absorber surface and the glass envelope is omitted.

\section{iv. Determination of optimal secondary-reflector height}

The relative height of the secondary reflector (the vertical position of the edge point) $\Delta h$ is actually an additional design parameter. A practical criterion to determine the optimal secondary-reflector height is the average distance between secondary surface and absorber. The shorter the average distance, the larger the average absorber acceptance angle - and thus, the more energy the absorber can intercept from the secondary reflection. 


\section{Optimization of a Secondary Reflector for the Hyperlight Energy Linear Fresnel Technology}

Here, a commercial linear Fresnel design under development is used as a case study. Hyperlight Energy developed a new type of linear Fresnel reflector [28]. It uses waterborne hollow plastic tubes to carry mini-size mirrors, as shown in Figure 6. A certain number of reflectors make up a basin. For a linear Fresnel collector module, there is one basin on each side of the receiver assembly fixed in the sky.
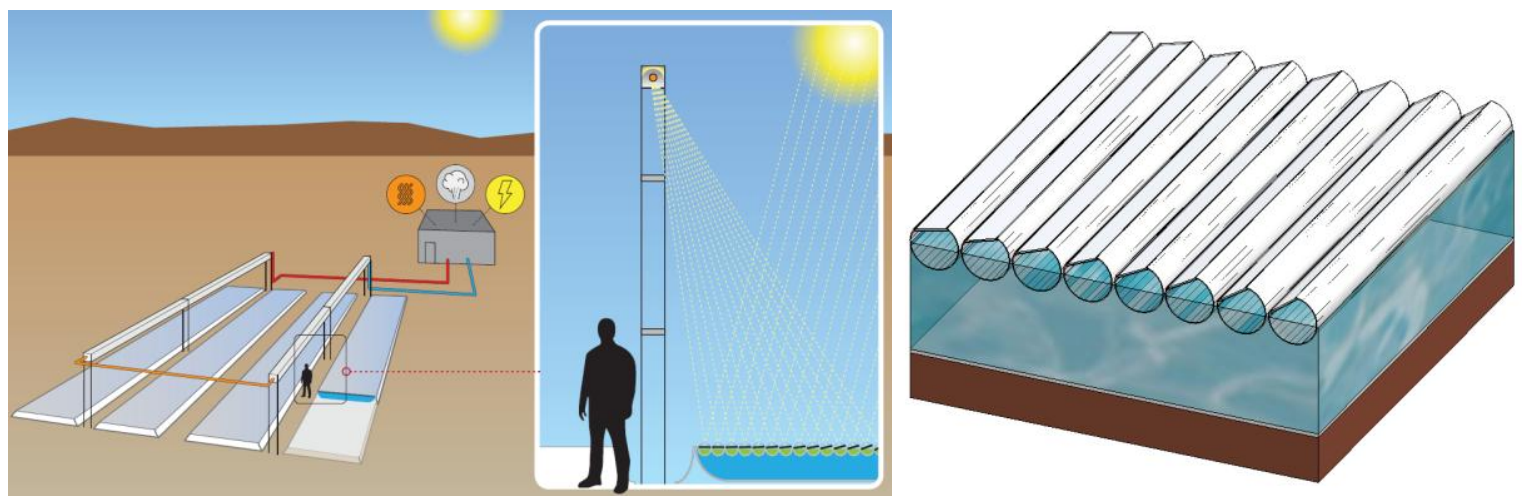

Figure 6: Illustration of Hyperlight Energy linear Fresnel reflectors.

\section{i. Configuration of Hyperlight Energy collector}

The geometrical and optical attributes of the current generation of Hyperlight Energy reflectors is summarized in Table 1 and Table 2.

Table 1: Geometrical specifications of the Hyperlight Energy reflector assembly.

\begin{tabular}{|l|l|}
\hline Total collector width $(\mathrm{m})$ & 12.8 \\
\hline $\begin{array}{l}\text { Total collector effective width }\left(\mathrm{W}_{\text {field }}, \mathrm{m}\right) \\
\text { measured from mirror edges at the two ends }\end{array}$ & 12.5 \\
\hline Net reflector aperture width $\left(\mathrm{W}_{\text {primary }}, \mathrm{m}\right)$ & 10.5 \\
\hline No. of basins along width & 2 \\
\hline Basin width $(\mathrm{m})$ & 6.1 \\
\hline Basin length $(\mathrm{m})$ & 15.7 \\
\hline Basin spacing along width $(\mathrm{m})$ & 1.0 \\
\hline Basin spacing along length $(\mathrm{m})$ & 0.67 \\
\hline No. of reflectors per basin & 50 \\
\hline Reflector width $(\mathrm{m})$ & 0.105 \\
\hline Reflector spacing $(\mathrm{m})$ & 0.115 \\
\hline $\begin{array}{l}\text { Position of innermost reflector relative to } \\
\text { the inner edge }(\mathrm{m})\end{array}$ & 0.15 \\
\hline $\begin{array}{l}\text { Position of outermost reflector relative to } \\
\text { the inner edge }(\mathrm{m})\end{array}$ & 5.9 \\
\hline Receiver assembly & \\
\hline Absorber tube height $(\mathrm{m})$ & 7 \\
\hline Absorber tube diameter $(\mathrm{m})$ & 0.09 \\
\hline
\end{tabular}


Table 2: Optical properties of the Hyperlight Energy reflector assembly based on preliminary measurements or vendor's specifications.

\begin{tabular}{|c|l|}
\hline Primary reflector & \\
\hline Reflectance of primary reflector & 0.94 \\
\hline $\begin{array}{l}\text { RMS of specularity (Gaussian, } \\
\text { mrad) }\end{array}$ & 1.5 \\
\hline $\begin{array}{l}\text { RMS of slope error and tracking } \\
\text { error(Gaussian, mrad) }\end{array}$ & 3 \\
\hline Absorber tube & \\
\hline Transmittance of glass envelope & 0.97 \\
\hline Absorptance of surface coating & 0.96 \\
\hline $\begin{array}{l}\text { Vertical position error range (, } \\
\text { mm) }\end{array}$ & {$[0,30]$} \\
\hline Secondary reflector & 0.94 \\
\hline Reflectance of primary reflector & 1.5 \\
\hline Specularity (Gaussian, mrad) & 2 \\
\hline Slope error (Gaussian, mrad) & {$[0,20]$} \\
\hline Vertical position error range (mm) & \\
\hline
\end{tabular}

\section{ii. Determination of receiver aperture}

Here is a simple illustration of receiver assembly aperture determination. By using the beam-spread RMS estimation Eq. (1) and if the receiver position error is neglected, the receiver assembly aperture can be obtained:

$$
\begin{gathered}
\sigma_{\text {total-reflector }}^{2}=\sigma_{\text {sun }}^{2}+\sigma_{\text {specularity }}^{2}+4 \cdot \sigma_{\text {slope }+ \text { track }}^{2} \\
=2.8^{2}+1.5^{2}+4 \times 3.0^{2} \cong 6.8^{2} .
\end{gathered}
$$

Here, the sun shape is assumed to have a circumsolar ratio (CSR) of $10 \%$ and it has a RMS of about 2.8 mrad [29]; the mirror specularity, slope error, and tracking error are referred to in Table 2.

$\sigma_{\text {total-reflector }}$ is the RMS of the beam spread after accounting for all reflector errors.

Assuming the sun shape and system optical errors follow a Gaussian distribution, then the required acceptance angle to capture $95 \%$ of reflected power at the outer edge of the outermost mirror $n$ at the normal incidence will be:

$$
\beta_{n}^{p r}=4 \times \sigma_{\text {total-reflector }}=27.2 \mathrm{mrad}
$$

Here, $\beta_{n}^{p r}$ is illustrated in Figure 1(b).

The required receiver aperture can be calculated as:

$$
\begin{aligned}
a_{2 n d} \cong l_{n} \cdot \sin & \left(\beta_{n}^{p r}\right)+w_{n} \cdot \cos \left(\tau_{n}\right) \\
& =\sqrt{\left[7^{2}+\left(\frac{12.5}{2}-\frac{0.105}{2}\right)^{2}\right]} \times \sin (0.0272)+0.105 \times \cos (0.36) \\
& \cong 0.35 \mathrm{~m}
\end{aligned}
$$




\section{iii. Selection of the absorber tube}

The geometric concentration ratio of a linear Fresnel collector can be defined as:

$$
C=\frac{W_{\text {primary }}}{D_{\text {absorber }}}
$$

The higher the concentration, the less the absorber surface per primary mirror area-which leads to less heat loss, thus increasing the collector thermal efficiency. If the absorber-tube diameter is $0.09 \mathrm{~m}$, the geometric concentration ratio will be about 117 for the Hyperlight Energy configuration. With such a high concentration ratio, if non-evacuated absorbers are used, the secondary reflector may be exposed to relatively high temperature. This may lead to very limited choices in the secondary reflector and increased cost. Thus, an evacuated absorber is selected to minimize the secondary-reflector surface temperature.

For evacuated absorbers in the market, the largest available size is about $0.09 \mathrm{~m}$ from parabolic trough collector products. Due to the elongated optical path (from primary reflector to receiver assembly) for linear Fresnel when compared with parabolic trough, the vacuum absorber with a diameter of $0.09 \mathrm{~m}$ is selected. For the identified receiver, the glass envelope has an outer diameter of $0.12 \mathrm{~m}$.

\section{iv. Optimized secondary-reflector profile}

Following the optimization methodology in Section III, an optimized secondary-reflector shape can be determined based on the Hyperlight Energy collector configuration. One example is illustrated in Figure 7.

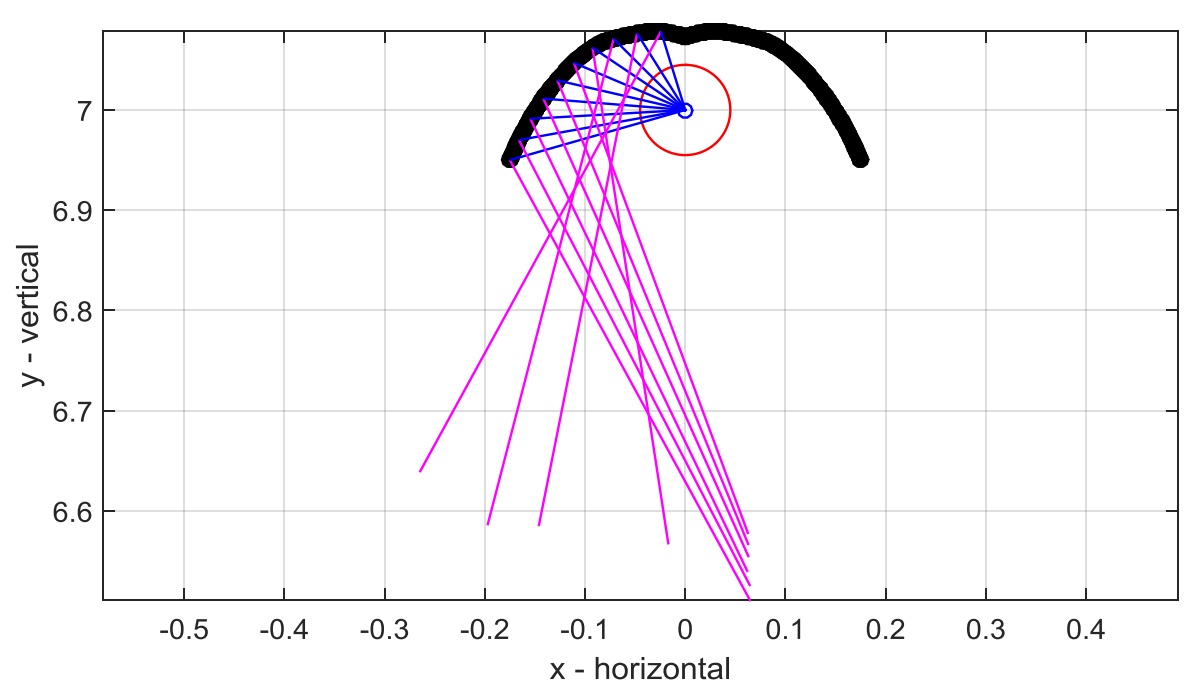

Figure 7: Optimized secondary-reflector shape for the Hyperlight Energy collector using the absorber with a diameter of $0.09 \mathrm{~m}$. The glass envelope is omitted.

The performance of a secondary reflector can be characterized by the following metric, the secondary intercept factor:

$$
\gamma_{2 n d}\left(\theta_{\perp}, \theta_{\|}\right)=\frac{\text { Power intercepted by absorber }}{\text { Power intercepted by secondary reflector aperture }}
$$




$$
\cong \gamma_{2 n d}^{t}\left(\theta_{\perp}\right) \cdot \gamma_{2 n d}^{l}\left(\theta_{\|}\right)
$$

For a selected absorber, the higher the intercept factor, the better the efficiency of the collector performance. Here, $\theta_{\perp}$ and $\theta_{\|}$are the transversal and longitudinal incidence angle, respectively [21]. The secondary intercept factor is decomposed as two individual secondary intercept factors $\left(\gamma_{2 n d}^{t}\right.$ and $\left.\gamma_{2 n d}^{l}\right)$ along transversal and longitudinal directions for mathematical simplification.
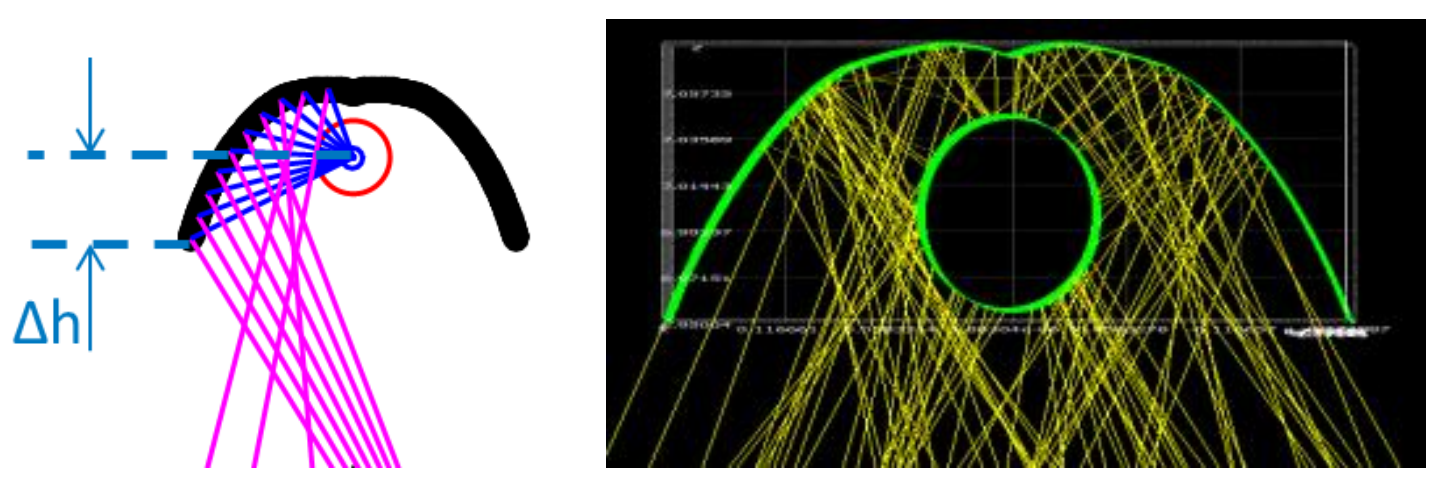

Figure 8: Definition of relative edge height as the secondary-reflector profile design parameter (left) and a snapshot of a SolTrace simulation (right).

In the Hyperlight Energy collector configuration, the absorber position is fixed at 7 meters and the secondary reflector has one additional design parameter - the relative edge height $\Delta h$, which is defined as the relative vertical distance between the absorber center and the secondary edge point, as shown in Figure 8. To calculate the impact of the relative secondary reflector height to the secondary intercept factor, a ray-trace software - SolTrace - is employed to perform optical simulation of the Hyperlight Energy collector. SolTrace is a software tool that the National Renewable Energy Laboratory (NREL) has developed to model and analyze the optical performance of CSP collectors [30, 31]. It uses the MonteCarlo algorithm to simulate the sun shape and can efficiently account for effects of various system optical errors. A sophisticated SolTrace model is developed to accommodate the Hyperlight Energy collector configuration (geometry and optics) and simulate the optical performance of derived secondary-reflector profiles. Figure 9 shows the secondary intercept factor at normal incidence with varying relative edge height of secondary reflectors. It can be seen that the secondary intercept factor becomes highest at the relative edge height of $0.05 \mathrm{~m}$, but the secondary intercept factor is higher than $90 \%$ in most cases. To understand the rationale behind this fact, the average surface distance to the absorber center is also plotted as a function of relative edge height. The maximum secondary intercept factor corresponds to the minimum average distance. The shorter the average distance, the larger the absorber acceptance angle to the secondary reflector. 

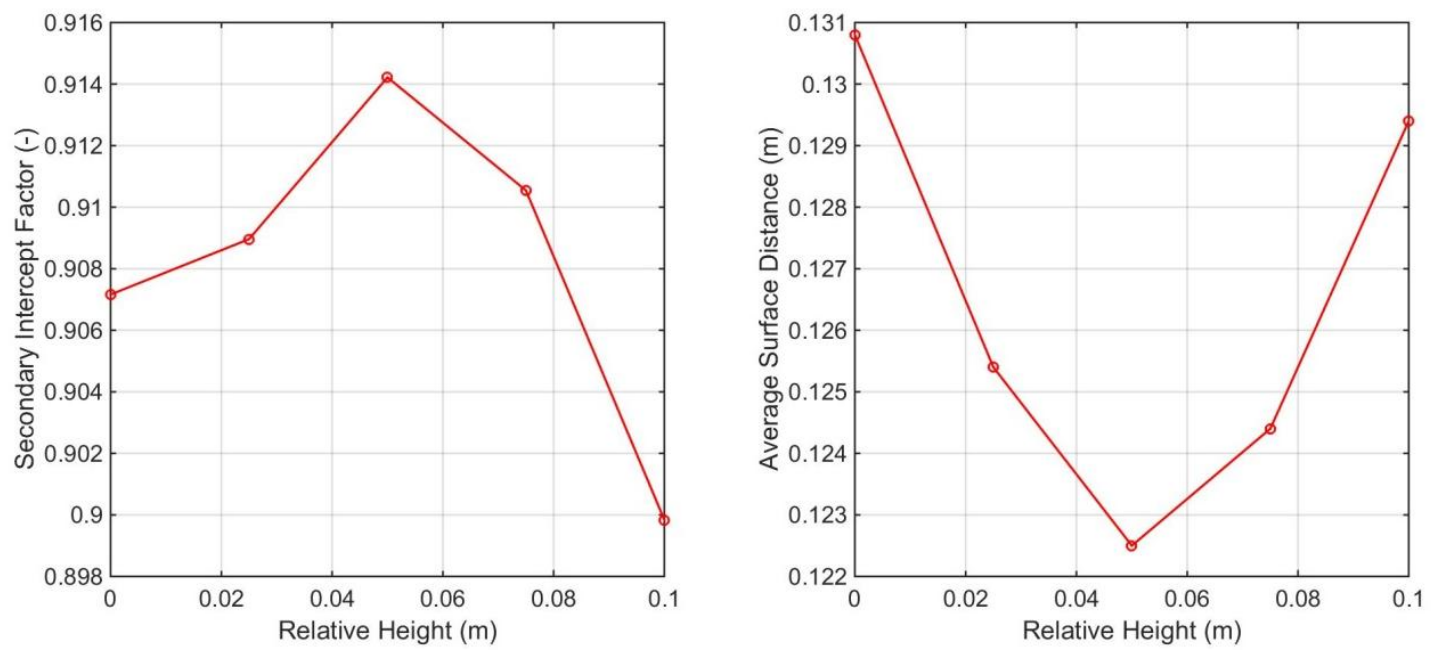

Figure 9: Secondary intercept factor (left) and average surface distance of the secondary-reflector profiles to the absorber center as a function of relative edge height.

After the relative edge height is optimized, the final optimal secondary reflector is determined as well. Its secondary intercept factor at different sun positions is then calculated through SolTrace models. In Figure 10, the secondary intercept factor of the optimal secondary-reflector profile is plotted as a function of both transversal and longitudinal incidence angles. Note that the secondary-reflector reflectance is assumed to be 0.94 and has been accounted for in the SolTrace models. From the plot, the secondary intercept factor stays between 0.91 and 0.92 for the whole range of transversal incidence; and with the increasing longitudinal incidence angle, the secondary intercept factor only starts to drop significantly at incidence angles higher than $70^{\circ}$. It is evident that the derived secondary-reflector profile is able to remain at a consistently high performance in the majority of sun positions during a year and exhibit its robustness with varying incidence angles. 


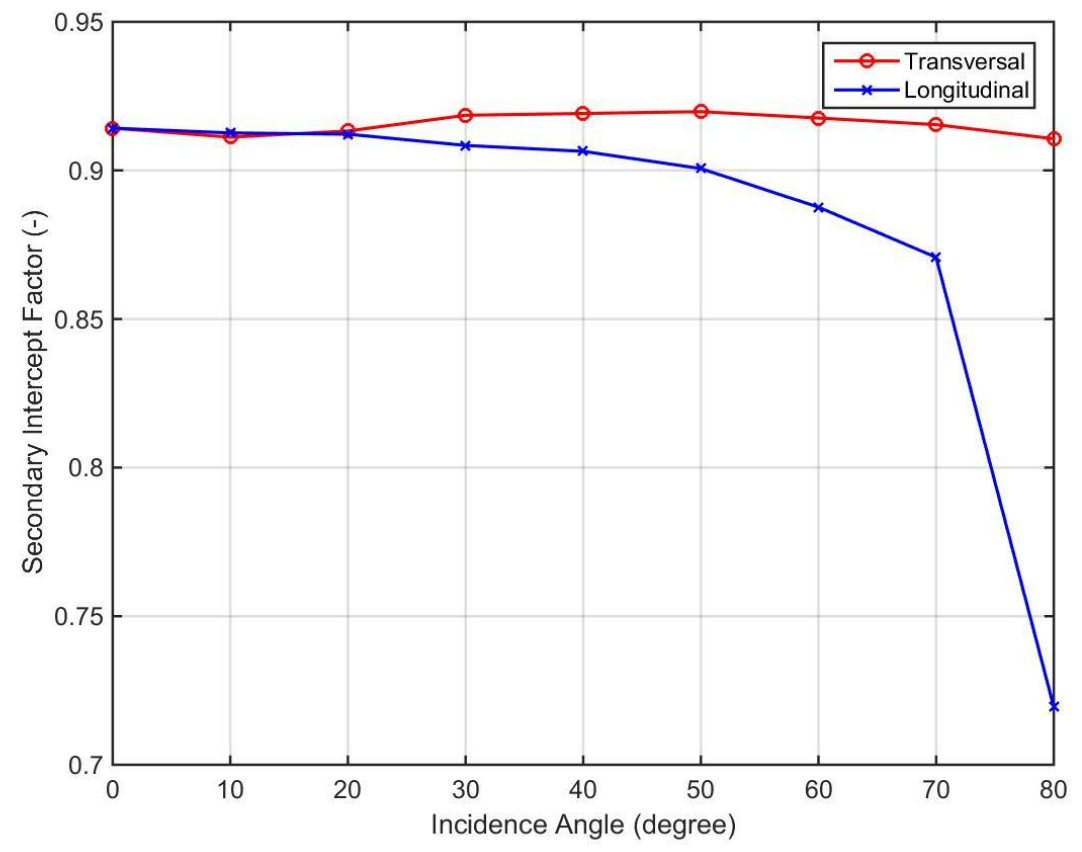

Figure 10: Secondary intercept factor as a function of incidence angle to characterize the performance of the optimal secondary-reflector profile. The secondary-reflector reflectance equal to 0.94 is assumed in the ray-trace simulations.

\section{v. Projected collector performance}

The collector optical efficiency can be defined as:

$$
\begin{aligned}
\eta(\theta)=\rho \cdot \tau \cdot \alpha \cdot & \gamma_{1 s t}\left(\theta_{\perp}, \theta_{\|}\right) \cdot \gamma_{2 n d}\left(\theta_{\perp}, \theta_{\|}\right)=\eta_{o} \cdot \operatorname{IAM}\left(\theta_{\perp}, \theta_{\|}\right) \\
& \cong \eta_{o} \cdot \operatorname{IAM}^{t}\left(\theta_{\perp}\right) \cdot \operatorname{IAM}^{l}\left(\theta_{\|}\right)
\end{aligned}
$$

Here, $\rho$ is the primary mirror reflectance, $\tau$ is the absorber glass-envelope transmittance, and $\alpha$ is the average absorber coating absorptance. $\eta_{o}$ is the nominal optical efficiency and IAM is the incidence angle modifier, which can be approximated by decomposing IAM into two incidence angle modifiers (IAM ${ }^{t}$ and $I A M^{l}$ ) along transversal and longitudinal directions. $\gamma_{1 s t}$ is the primary intercept factor accounting for the cosine loss, shading and blocking effect, and intercept accuracy of primary reflector array with respect to the secondary-reflector aperture:

$$
\gamma_{1 s t}\left(\theta_{\perp}, \theta_{\|}\right)=\frac{\text { Power intercepted by secondary reflector aperture }}{\text { Power intercepted by the entire primary reflector aperture }}
$$

By employing SolTrace models, the optical performance of the Hyperlight Energy collector with the optimal secondary profile can be calculated. In Figure 11, the incidence angle modifiers are plotted. The nominal optical efficiency at normal incidence is 0.699 from the SolTrace simulations. 


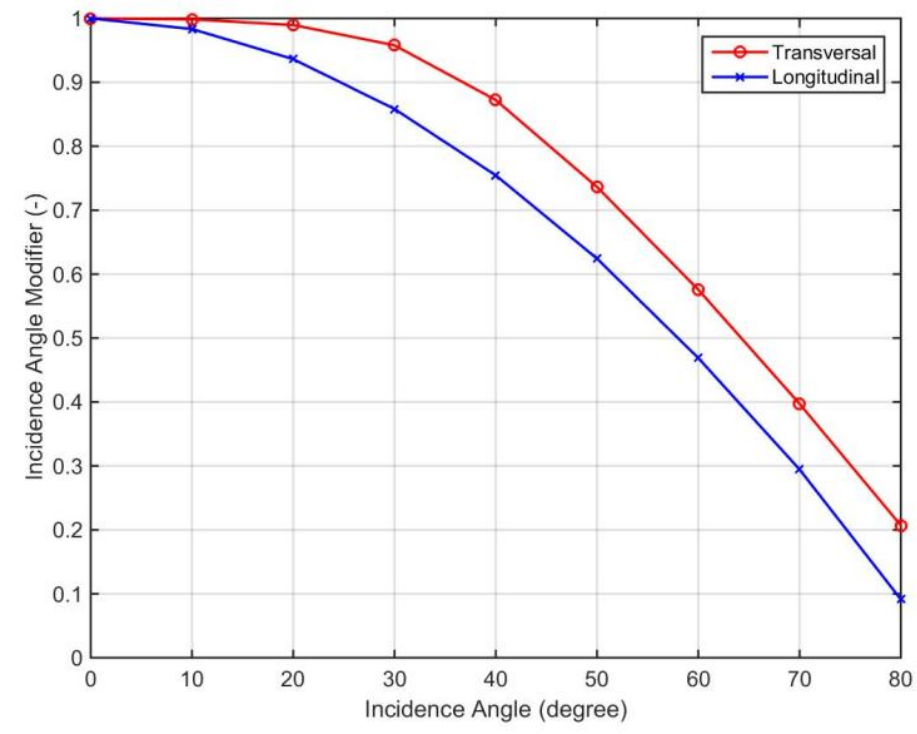

Figure 11: Incidence angle modifier as a function of incidence angle for Hyperlight Energy collector with the derived optimal secondary reflector. The nominal optical efficiency is calculated as 0.699.

The optical loss of a linear Fresnel collector comes from four sources:

- Loss due to component optical properties. This is the effect of primary-reflector reflectance, absorber absorptance, and absorber-envelope transmittance;

- Secondary-reflector intercept loss. This accounts for the secondary-reflector reflectance and the fact that some reflected light by the secondary reflector will miss the absorber;

- Primary intercept loss excluding cosine loss. This accounts for shading and blocking of primaryreflector array and the fact that some reflected light by the primary reflector will miss the secondary-reflector aperture; and

- Primary intercept loss - cosine loss. This accounts for the cosine loss of the primary-reflector array.

The relative contribution of each individual optical loss source is plotted for varying transversal and longitudinal incidence angles in Figure 12. For the increasing in both incidence angles, the optical loss due to component optical properties and secondary intercept loss roughly remains constant and this again illustrates the robustness of the optimized secondary profile. The cosine loss of primary reflectors increases with increasing transversal or longitudinal incidence angle, but this is due to the nature of the linear Fresnel configuration and cannot be further reduced at a significant level. The primary-reflector intercept loss excluding cosine loss has a substantial contribution to the collector performance as shown in the figure. Particularly for the increasing transversal incidence angle, the primary optical loss starts to dominate the overall collector optical loss. This portion of primary intercept loss comes primarily from shading and blocking and largely depends on the collector geometrical configuration such as primaryreflector spacing and the receiver-assembly height. With increasing primary spacing, the shading and blocking will decrease, but more reflected sun rays will miss the receiver assembly due to the increasing optical path. This indicates that there exists an optimal primary spacing and a receiver assembly height that will produce the highest collector performance. At the same time, the determination of a set of optimal collector geometrical parameters will also require accounting for associated economics of the 
overall linear Fresnel collector depending on specific applications. Linear Fresnel collector optimization will be a separate topic. The stacked bar plots with different optical-loss sources will shed light on future collector optimization.
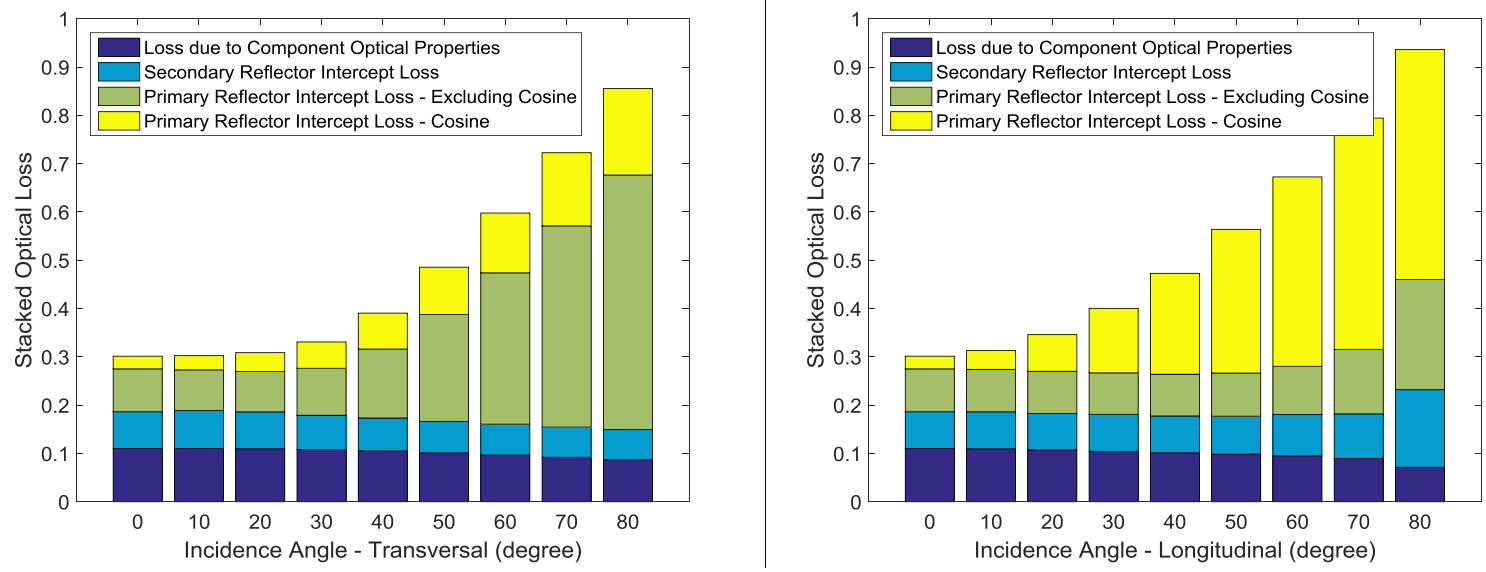

Figure 12: Stacked optical loss based on individual error sources for varying incidence angle — transversal (left) and longitudinal (right).

\section{Discussion and Conclusions}

In this work, an adaptive optimization methodology for a secondary-reflector profile is developed and demonstrated by applying it to an existing novel linear Fresnel design. Instead of starting from a set of parameters defining the secondary-reflector profile, the presented work establishes a complete procedure to efficiently derive an optimal secondary profile able to reflect the maximum amount of power to the absorber. An industrial linear Fresnel collector design is used to illustrate the feasibility and robustness of the adaptive optimization method. It is shown that the derived secondary-reflector profile can achieve an efficiency of more than $90 \%$ for a wide range of transversal and longitudinal incidence angles.

The critical step in the adaptive optimization method is the determination of principal incidence at each secondary surface point. The work provides an accurate but straightforward approach to identify principal incidence. Throughout various optimal secondary profiles, principal incidence moves from one side of the primary-reflector field to the other. This indicates that the secondary profile is dramatically different from a parabola, but able to achieve highest optical performance.

The developed adaptive optimization method for secondary reflectors can be applied to different types of linear Fresnel reflectors using primary reflectors of any shape (including flat, circular, and parabolic shapes) and one or multiple absorbers. It can also be applied to other types of solar collectors (including power tower and dish/engine technologies) when a secondary reflector is needed. NREL has submitted a patent application on the proposed adaptive optimization method.

\section{Acknowledgments}

NREL is a national laboratory of the U.S. Department of Energy, Office of Energy Efficiency and Renewable Energy, operated by the Alliance for Sustainable Energy, LLC. This work was supported by Southern California Gas Company as part of an award under PON 14-303 released by the California Energy Commission (CEC) Electric Program Investment Charge (EPIC) program, through a CEC/EPIC SubTier Funds-In agreement with NREL. 
The U.S. Government retains and the publisher, by accepting the article for publication, acknowledges that the U.S. Government retains a nonexclusive, paid up, irrevocable, worldwide license to publish or reproduce the published form of this work, or allow others to do so, for U.S. Government purposes.

\section{References}

1. Zhu, G., et al., History, Current-State and Future of Linear Fresnel Concentrating Solar Collectors. Solar Energy, 2014. 103: p. 639-652.

2. Mills, D., Adances in Solar Thermal Electricity Technology. Solar Energy, 2004. 76: p. 19-31.

3. Mills, D. and G. Morrison, Compact Linear Fresnel Reflector Solar Thermal Power Plants. Solar Energy, 2000. 68(3): p. 262-283.

4. Novatec Solar Available from: http://www.novatecsolar.com/.

5. Areva Solar. Available from: http://www.areva.com/EN/solar-220/areva-solar.html.

6. Brost, R. and G. Zhu. Design of a High-Temperature Molten Salt Linear Fresnel Collector. 2010; Available from: http://www1.eere.energy.gov/solar/review_meeting/pdfs/prm2010_skyfuel.pdf.

7. Brost, R. and G. Zhu, Commercial Development of an Advanced, High-Temperature, LinearFresnel Based Concentrating Solar Power Concept. 2009, SkyFuel: Prepared under DOE FOA No. DE-FC36-08GO18034.

8. Areva Solar, US Sandia Labs Join Forces for CLFR Molten-Salt Storage. Available from: http://www.solarnovus.com/index.php?option=com_content\&view=article\&id=5656:areva-solarus-sandia-labs-join-forces-for-clfr-molten-salt-storage\&catid=41:applications-technews\&Itemid $=245$.

9. Morin, G., et al., Molten Salt as a Heat Transfer Fluid in a Linear Fresnel Collector Commercial Application Backed by Demonstration, in SolarPACES 2014. 2014: Beijing, China.

10. Grena, R. and P. Tarquini, Solar Linear Fresnel Collector Using Molten Nitrates as Heat Transfer Fluid. Energy, 2011. 36: p. 1048-1056.

11. Singh, P.L., S. Ganesan, and G.C. Yadav, Performance Study Of A Linear Fresnel Concentrating Solar Device. Renewable Energy, 1999. 18: p. 409-416.

12. Industrial Solar. Available from: http://www.industrial-solar.de/CMS/en/.

13. Lai, Y., et al., Thermal Performance Analysis of Linear Fresnel Reflector Concentrator with a Compound Parabolic Cavity Absorber, in 2011 International Conference of ICMREE. 2011: Shanghai, China.

14. Singh, P.L., R.M. Sarviya, and J.L. Bhagoria, Thermal Performance of Linear Fresnel Reflecting Solar Concentrator With Trapezoidal Cavity Absorbers. Applied Energy, 2010. 87: p. 541-550.

15. Winston, R., J.C. Minano, and P. Benitez, Nonimaging Optics. 2005: Elsevier Academic Press.

16. Winston, R. and H. Hinterberger, Principles of Cylindrical Conentrators for Solar Energy. Solar Energy, 1975. 17.

17. Zhu, G., D. Kearney, and M. Mehos, On Characterization and Measurement of Average Solar Field Mirror Reflectance in Utility-Scale Concentrating Solar Power Plants. Solar Energy, 2014. 99: p. 185-202.

18. Nicodemus, F.E., et al., Geometrical Considerations and Nomenclature for Reflectance. 1977, National Bureau of Standards, Dpeartment of Commerce.

19. Gee, R., et al., An Improved Method for Characterizing Reflector Specularity for Parabolic Concentrators, in 16th SolarPACES, . 2010: Perpignan, France.

20. Zhu, G. and A. Lewandowski, A New Optical Evaluation Approach for Parabolic Trough Collectors: First-principle OPTical Intercept Calculation (FirstOPTIC). Journal of Solar Energy Engineering, 2012. 134.

21. Zhu, G., Development of an Analytical Optical Method for Linear Fresnel Collectors. Solar Energy, 2013. 94. 
22. Walzel, M.D., F.W. Lipps, and L. Vant-Hull, A Solar Flux Density Calculation for a Solar Tower Concentrator Using a Two-Dimensional Hermite Function Expansion. Solar Energy, 1977. 19(3): p. 239-253.

23. Rabl, A., Active Solar Collectors and Their Applications. 1985, New York: Oxford University Press.

24. Bendt, P., et al., Optical Analysis and Optimization of Line Focus Solar Collectors. 1979, SERI: Golden, CO, USA.

25. Biggs, N. and C.N. Vittitoe, The Helios Model for the Optical Behavior of Reflecting Solar Concentrators. 1976, Sandia National Laboratories: Albuquerque, NM.

26. Kistler, B., A user's manual for DELSOL3: A computer code for calculating the optical performance and optimal system design for solar thermal central receiver plants. 1986, Sandia Ntional Laboratories: Albuquerque, NM.

27. Ratzel;, A.C. and B.D. Boughton;, CIRCE.001: A Computer Code for Anaysis of Point-Focus Concentrators with Flat Targets. 1987, Sandia National Laboratories: Albuquerque, NM.

28. Hyperlight Energy. 2015; Available from: http://www.hyperlightenergy.com/

29. Neumann, A., et al., Representative Terrestrial Solar Brightness Profiles. Journal of Solar Energy Engineering, 2002. 124: p. 198-204.

30. Wendelin, T., SolTrace: A New Optical Modeling Tool for Concentrating Solar Optics, in 2003 International Solar Energy Conference, . 2003: Hawaii, USA.

31. SolTrace. Available from: http://www.nrel.gov/csp/soltrace.html. 